\title{
Les éléments de réponse \\ à la vitamine $D$, à l'acide rétinoïque et aux hormones thyroïdiennes
}

La réponse transcriptionnelle aux hormones stéroïdes et thyroïdiennes, à la vitamine $\mathrm{D}$ et à l'acide rétinoïque se fait via la fixation de complexes ligand/récepteur au niveau de séquences régulatrices des gènes.

Les récepteurs appartiennent tous ici à la même famille de protéines dont les similitudes maximales se retrouvent au niveau d'une région de fixation à des éléments d'ADN appelés HRE (hormone response elements). Tous ces HRE, spécifiques des différentes hormones, ont des séquences voisines, presque toujours palindromiques (c'est-à-dire identiques sur les deux brins d'ADN), comme cela est montré sur le Tableau I. Les deux derniers éléments de response à avoir été identifiés sont ceux de l'acide rétinoïque et de la vitamine D. Hugues de
Thé et Anne Dejean, du laboratoire de Pierre Tiollais à l'Institut Pasteur de Paris, en collaboration avec des chercheurs de l'EMBL (Heidelberg, RFA), viennent de caractériser l'élément de réponse à l'acide rétinoïque, dénommé RARE (retinoic acid response element), localisé immédiatement en amont de la boîte TATA du promoteur du gène codant pour le récepteur $\beta$ de ce même acide rétinoïque. Cet élément RARE rend compte de la stimulation de l'expression du gène du récepteur par le ligand. La caractéristique la plus intrigante de cette séquence est l'existence de deux répétitions directes (c'est-à-dire orientées dans le même sens) d'un hexanucléotide qui est du type de ceux constituant les hémipalindromes des autres HRE de cette famille. Rappelons -

Tableau 1

QUELQUES SÉQUENCES DE RÉPONSE AUX HORMONES

\begin{tabular}{|c|c|c|c|c|c|c|c|}
\hline \multicolumn{3}{|c|}{$G \quad G \quad T A C A$} & NNN & \multicolumn{3}{|c|}{$\begin{array}{llllll}T & G & T & T & C & T\end{array}$} & GRE, consensus \\
\hline G G & $\mathrm{T}$ & $C \stackrel{A}{\longrightarrow}$ & NNN & $\stackrel{T}{T}$ & A & $\mathrm{C} \mathrm{C}$ & ERE, consensus \\
\hline G G & $\mathrm{T}$ & $\stackrel{\mathrm{C}}{\rightarrow}$ & $\mathrm{N}$ & $\stackrel{T}{\leftarrow} G$ & A & C C & $\begin{array}{l}\text { TRE, palindrome } \\
\text { synthétique }\end{array}$ \\
\hline G G & $\mathrm{T}$ & $\mathrm{G} \stackrel{A}{\longrightarrow}$ & C & $T^{T} G$ & A & $\mathrm{C} \mathrm{C}$ & G G T G A VDRE ostéocalcine \\
\hline G G & T & 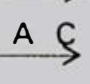 & GAAA & G T & $\mathrm{T} \mathrm{C}$ & $\stackrel{A}{\stackrel{C}{\longrightarrow}}$ & $\begin{array}{l}\text { RARE, gène } \beta \\
\text { du récepteur AR }\end{array}$ \\
\hline
\end{tabular}

GRE = glucocorticoid response element ; ERE = estrogen response element; TRE = thyroid hormone response element; VDRE = vitamin $D$ response element.

Les flèches indiquent la direction des répétitions : inversée pour les séquences palin dromiques, directe pour l'élément RARE. RARE = retinoic acid response element.

Observer que l'ERE et la TRE représentés ici ne diffèrent que par le nombre de base $N$ entre les deux hémipalindromes.

$m / s n^{\circ} 3$ vol. 6 , mars 90

ainsi que nous l'avons déjà signalé dans $\mathrm{m} / \mathrm{s}$ - que des TRE (thyroid hormone response element) peuvent aussi conférer la réponse à l'acide rétinoïque, si bien que des travaux complémentaires sont nécessaires pour préciser les rapports fonctionnels entre RARE et TRE, entre récepteurs des hormones thyroïdiennes et de l'acide rétinoïque. Comme indiqué dans une "brève " de $\mathrm{m} / \mathrm{s}$, ces deux types de récepteur pourraient coopérer via la formation de dimères hybrides dont il sera intéressant d'étudier l'effet lorsqu'ils se fixent sur des séquences RARE ou TRE. De même, une éventuelle fixation inhibitrice du produit de l'oncogène $v$-erbA sur l'élément RARE serait intéressante à étu$\operatorname{dier}\left(\mathrm{m} / \mathrm{s} n^{\circ} 8\right.$, vol. 5, p. 601)

L'élément de réponse à la vitamine $\mathrm{D}$ a également été récemment identifié en amont du gène d'une protéine de l'os, l'ostéocalcine, par une équipe australienne de Sidney [2]. La particularité de cette séquence est de comporter un double palindrome (Tableau I) et de chevaucher, probablement, une GRE (glucocorticoid response element) qui pourrait expliquer l'inhibition par les glucocorticoïdes de certains effets métaboliques de la vitamine $\mathrm{D}$ sur l'os.

A. $\mathbf{K}$.

1. De Thé $\mathrm{H}$, Vivanco-Ruiz MDM, Tiollais $\mathrm{P}$, Stunnenberg $\mathrm{H}$, Dejean A. Identification of a retinoic acid response element in the retinoic acid receptor $\beta$ gene. Nature $1990 ; 343$ : 177-80.

2. Morrison NA, Shine J, Fragonas JC, Verkest $\mathrm{V}$, McMenemy ML, Eisman JA. 1,25 dihydroxy-vitamin D-responsive element and glucocorticoid repression in the osteocalcin gene. Science 1990 ; 246 : 1158-61. 